\title{
Bulk and surface characterisation of micrometer-thick cobalt ferrite films grown by IR PLD
}

\author{
M. Sánchez-Arenillas ${ }^{\mathrm{a}}$, M. Oujja ${ }^{\mathrm{a}}$, F. Moutinho ${ }^{\mathrm{a}}$, J. de la Figuera ${ }^{\mathrm{a}}$, M.V. Cañamares ${ }^{\mathrm{b}}$, A. Quesada ${ }^{\mathrm{c}}$, M. Castillejo ${ }^{\mathrm{a}}$, \\ J.F. Marco ${ }^{\mathrm{a}}$ \\ aInstituto de Quimica Física “Rocasolano”, CSIC, Madrid E-28006, Spain \\ ${ }^{b}$ Instituto de Estructura de la Materia, CSIC, Madrid E-28006, Spain \\ ${ }^{c}$ Instituto de Cerámica y Vidrio, CSIC, Madrid E-28049, Spain
}

\begin{abstract}
We have studied micrometer-thick cobalt ferrite films deposited on Si (100) single crystal substrates by nanosecond pulsed laser deposition at $1064 \mathrm{~nm}$. The thickness of the deposited films $(1.3 \mu \mathrm{m})$ was monitored by AFM. The chemical and structural characterisation of the films was carried out by Raman spectroscopy and transmission Mössbauer spectroscopy at 300 and $26 \mathrm{~K}$. For comparison purposes, transmission Mössbauer data at these two temperatures were also recorded from a commercial cobalt ferrite powder and the home-made target used to grow the films. The surface characterisation was performed by X-Ray Photoelectron Spectroscopy (XPS) and Integral Low Energy Electron Spectroscopy (ILEEMS). XPS showed Co and Fe to be present as $\mathrm{Co}^{2+}$ and $\mathrm{Fe}^{3+}$, as expected for cobalt ferrite. The Raman spectra showed the lines characteristic of cobalt ferrite. The Mössbauer spectra, both in the transmission and backscattering modes, were fitted to two sextets whose hyperfine parameters are in good agreement with those expected from $\mathrm{Fe}^{3+}$ cations occupying the tetrahedral and octahedral sites in the spinel-related structure. No significant differences were observed in the relative areas of the two sextets in the transmission and ILEEMS spectra, suggesting that the cation distribution at the surface and the bulk are not too different. However, the relative areas of the two components changed drastically with temperature both in the spectra of the films as in those recorded from the cobalt ferrite standards. We discuss the possible origin of the evolution with temperature of those relative areas.
\end{abstract}

\section{Introduction}

Ferrites are receiving an increasing interest due to their wealth of magnetic and electronic properties and also because they are being used in many technological applications such as spin filters, permanent magnets, resistive switching and magnetoelectronic coupling devices, microwave absorbers, hyperthermia, catalysts and chemical sensors [1, 2, 3]. In particular, cobalt ferrite $\left(\mathrm{CoFe}_{2} \mathrm{O}_{4}\right)$ has been widely used as electronic device, ferrofluid or high density information storage. Cobalt ferrite (CFO) is a mixed iron (III) and cobalt (II) oxide with a spinel-related structure. It is a cubic structure centred on the faces of oxygen anions. Ideally, half of the Fe(III) ions occupies the tetrahedral sites and the other half, together with $\mathrm{Co}(\mathrm{II})$ ions, the octahedral sites. Their electronic and magnetic properties

Email address: msanchezeigfr.csic.es (M. Sánchez-Arenillas)

Preprint submitted to Elsevier depend on the cation distribution. Cobalt ferrite is particularly interesting due to its high coercive field, high Curie temperature, large magnetocrystaline anisotropy and moderate saturation magnetization $[4,5,6,7,8]$. However, despite its technological importance, aspects about its electronic and magnetic properties must still be understood.

Some groups have studied how the magnetic properties of cobalt ferrite nanoparticles change with the particle size and CFO thin films have been grown by various methods, such as molecular beam epitaxy, sputtering, ion-beam deposition and sol-gel processing, trying to control parameters like grain size, film thickness, type of substrate, substrate temperature or lattice strain $[6,9,10,11]$.

PLD is a versatile technique for the growth of thin films allowing the deposition of various materials such as ferroelectrics, polymers, semiconductors or metal oxide materials. PLD is more versatile than other film growth techniques because the choice of the laser wave-

April 10, 2019 
length and other growth parameters allow controlling the size, shape and crystalline phase of the films and of the nanodeposits. [4, 12, 13, 14].

In a previous work [4] we have investigated cobalt ferrite PLD films of nanometer thickness deposited on $\mathrm{Si}$ wafers and have determined the best conditions to grow well-crystallized films (which corresponded with a substrate temperature of $770 \mathrm{~K}$ and a laser IR wavelength of $1064 \mathrm{~nm})$. In this work we want to extend those studies to thicker films (in the micrometer range) produced with those "ideal" deposition parameters but using much longer deposition times in order to investigate the influence of the deposition time on the chemical and structural characteristics of the films and, in particular, the possible differences in the surface and bulk cation distributions. We have carried out the surface and bulk characterisation of the deposited materials by Raman spectroscopy, X-ray photoelectron spectroscopy (XPS), transmission Mössbauer spectroscopy at two different temperatures (RT and $26 \mathrm{~K}$ ) and integral lowenergy electron Mössbauer spectroscopy (ILEEMS) at room temperature. For comparison purposes transmission Mössbauer spectra have been also recorded from a commercial cobalt ferrite powder and the home-made target used to grow the films by PLD.

\section{Experimental Methods}

The cobalt ferrite (CFO) target used in this study was synthesized following a sol-gel process using the Co and $\mathrm{Fe}$ nitrates $\mathrm{Co}\left(\mathrm{NO}_{3}\right)_{2} \cdot 6 \mathrm{H}_{2} \mathrm{O}$ and $\mathrm{Fe}\left(\mathrm{NO}_{3}\right)_{2} \cdot 9 \mathrm{H}_{2} \mathrm{O}$. The nitrates were dissolved in the 1:2 stoichiometric ratio in deionized water. Citric acid was subsequently added to the solution, which was then stirred at $333 \mathrm{~K}$ for $1 \mathrm{~h}$. The gel was dried in a hot air oven for $24 \mathrm{~h}$. The precursor obtained was calcined for $1 \mathrm{~h}$ at $673 \mathrm{~K}$. The obtained powders were sieved $\leq 63 \mu \mathrm{m}$ and colduniaxially pressed at $55 \mathrm{MPa}$ into disks of $15 \mathrm{~mm}$ of diameter and $2 \mathrm{~mm}$ of thickness. The targets were finally sintered in air at $1473 \mathrm{~K}$ for $2 \mathrm{~h}$, employing a 5 $\mathrm{K} /$ min warming up rate. Also, a commercial CFO powder from EVOCHEM Advanced Materials GmbH, was characterized for comparison.

The deposits were grown in a PLD system consisting of a stainless-steel vacuum chamber pumped down to $2 \cdot 10^{-4} \mathrm{~Pa}$ by a turbo-molecular pump $[4,13]$. The targets were placed on a rotating sample holder and ablated with a Q-switched Nd:YAG laser (FWHM 15 $\mathrm{ns}, 10 \mathrm{~Hz})$ at the excitation wavelength of $1064 \mathrm{~nm}$. The laser beam was focused onto the target at $45^{\circ}$ to a spot area of $0.14 \mathrm{~mm}^{2}$. The fluence (laser pulse energy per unit area) used for the fabrication of the de-

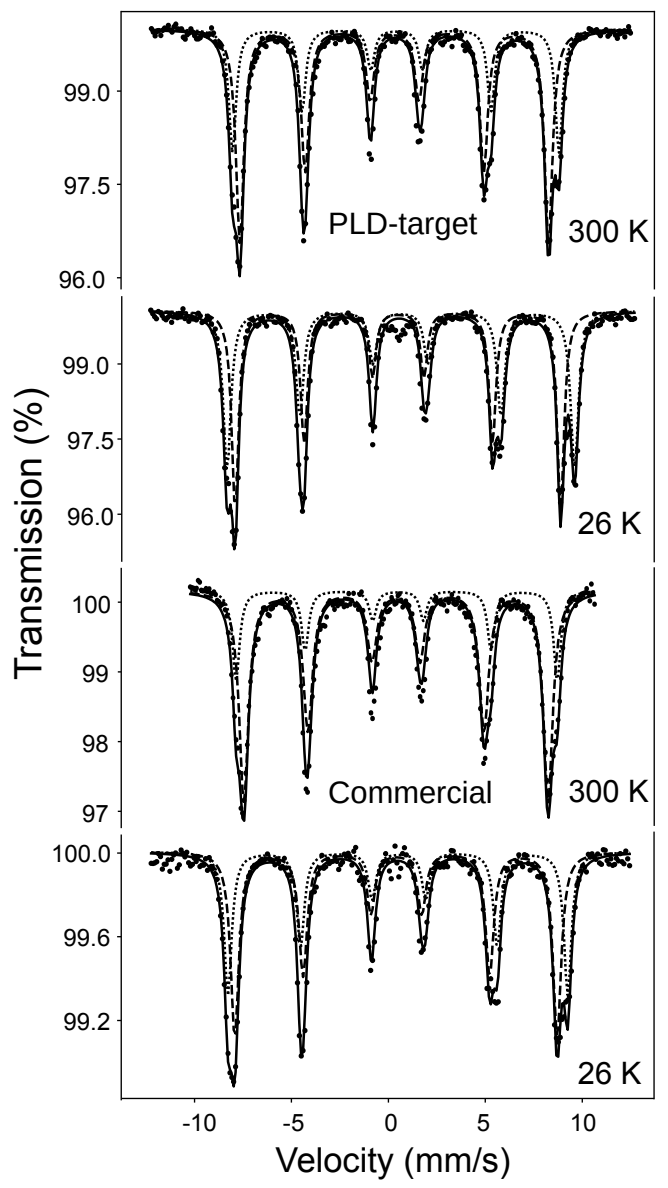

Figure 1: Transmission Mössbauer spectra of powder cobalt ferrite samples at different temperatures. Top: PLD target. Bottom: Commercial powder. Two sextet components, corresponding to $\mathrm{Fe}^{3+}$ in tetrahedral (dashed line) and octahedral (dotted line) sites, respectively, are shown.

posits was fixed to a value corresponding to 8 times the ablation threshold fluence. The threshold fluence was determined by measuring the minimum single pulse energy necessary to yield a luminous plume as detected by eye and the diameter of the irradiated region by the print left on an unplasticized polyvinyl chloride sheet. Based on the obtained ablation threshold, the used irradiation fluence was $4 \mathrm{~J} \mathrm{~cm}^{-2}$. Substrates $20 \mathrm{~mm} \times$ $20 \mathrm{~mm}$ in size of Si (100)(ACM company, France) were used for film growth and were ultrasonically degreased in acetone and methanol for $10 \mathrm{~min}$ prior to use. The substrates were mounted on a heating element that allows operation at a temperature of $770 \mathrm{~K}$, as measured by a thermocouple. They were placed in front of the cobalt ferrite target at a distance of $40 \mathrm{~mm}$. Deposits were grown by delivering 288.000 pulses to the target 


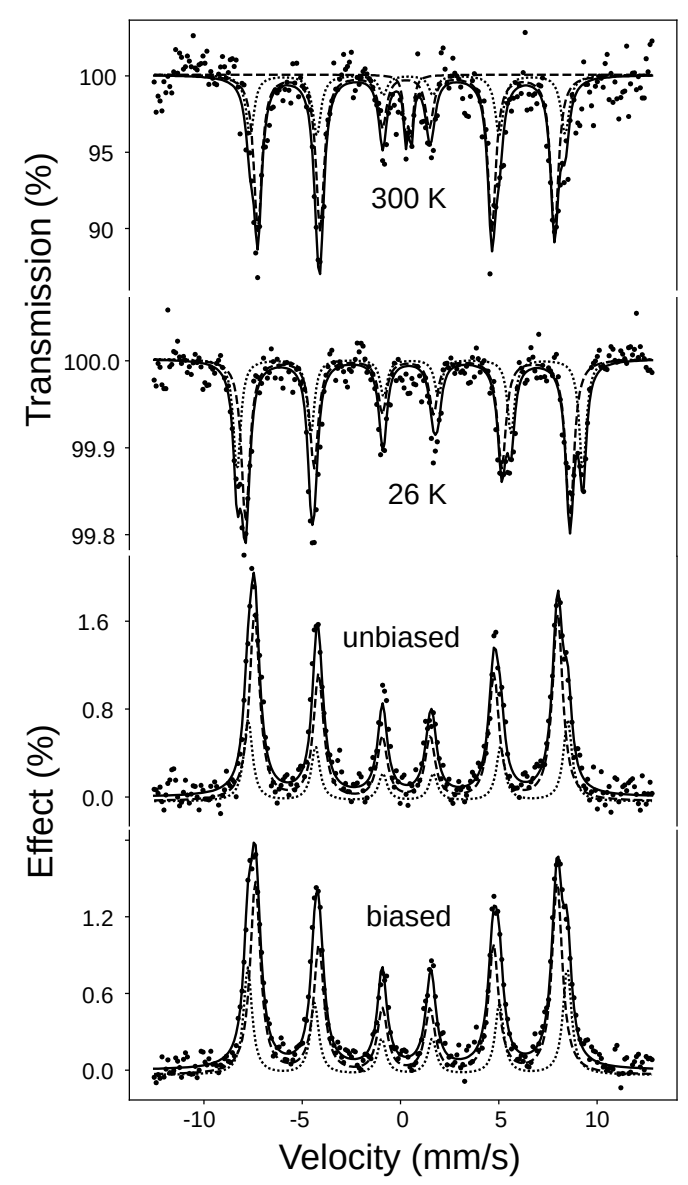

Figure 2: Mössbauer spectra of the cobalt ferrite film. Top: Mössbauer spectra acquired in the transmission mode at different temperatures. Bottom: Mössbauer spectra acquired in the ILEEMS. Two sextet components, corresponding to $\mathrm{Fe}^{3+}$ in tetrahedral (dashed line) and octahedral (dotted line) sites, respectively, are shown. An additional small component has been included in the fit of the film at room temperature for the transmission Mössbauer spectrum.

(resulting in a deposition time of around $8 \mathrm{~h}$ ).

The thickness of the cobalt ferrite film was examined by Atomic Force Microscopy (AFM, Molecular Imaging) in tapping mode. The elemental composition and the oxidation state of the surface cations were determined by X-ray Photoelectron Spectroscopy (XPS). The XPS spectra were recorded with a SPECS Phoibos150 hemispherical electron energy analyzer under a base pressure of $4 \cdot 10^{-10}$ torr using $\mathrm{Al} \mathrm{K} \alpha$ radiation. Constant pass energies of 100 and $20 \mathrm{eV}$ were used to record the wide scan and narrow scan spectra, respectively. The energy scale was referenced to the binding energy (BE) of the $\mathrm{C} 1 \mathrm{~s}$ core level of the adventitious contamination layer which was set at $285.0 \mathrm{eV}$.

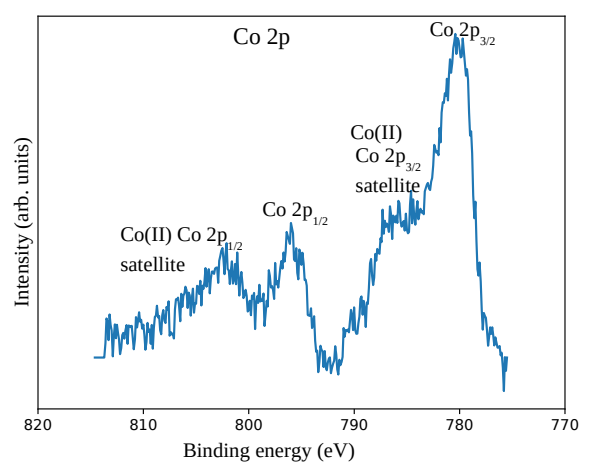

Figure 3: Co 2p XPS spectrum recorded from the film deposited on $\mathrm{Si}(100)$ by PLD at $1064 \mathrm{~nm}$

The Mössbauer spectra were measured in the conventional transmission mode in a helium closed-cycle cryorefrigerator using a ${ }^{57} \mathrm{Co}(\mathrm{Rh})$ source and a triangular drive waveform. Spectra were recorded at $300 \mathrm{~K}$ and $26 \mathrm{~K}$. The velocity scale was calibrated using a $\alpha-\mathrm{Fe}$ foil (6 $\mu \mathrm{m}$ thick) and the isomer shifts were referred to the centroid of the $\alpha$-Fe spectrum at room temperature. All the spectra were computer fitted.

${ }^{57} \mathrm{Fe}$ ILEEMS is a non-destructive technique that has been used to study the structural and magnetic properties of the surface of iron based materials. The theoretical and methodological bases of this technique have been reviewed elsewhere [15]. In this technique a channeltron electron multiplier in high or ultra high vacuum is used as the electron detector. In our particular experimental configuration, it is placed in front of the sample which is oriented $45^{\circ}$ upwards with respect to the incidence direction of $\gamma$ rays emitted by the ${ }^{57} \mathrm{Co}$ source. The $50 \mathrm{mCi}{ }^{57} \mathrm{Co}(\mathrm{Rh})$ source is mounted on a conventional mechanical drive and kept outside the vacuum chamber within a lead lined stainless steel tube, welded to a $\mathrm{CF}$ flange, that ends in a Be/Plexiglass window which lets in the $14.4 \mathrm{KeV} \gamma$ rays while filtering out the $6.4 \mathrm{KeV} \mathrm{Fe}(\mathrm{K} \alpha) \mathrm{X}$-Rays. The windowto-sample and channeltron-to-sample distances are approximately $5-6 \mathrm{~cm}$. The channeltron electron multiplier used has an entrance cone of $19 \mathrm{~mm}$ diameter and is operated in the pulse counting mode. Mössbauer backscattering spectra were acquired using a triangle velocity mode and a conventional pulse counting electronics, consisting of the biasing power supplies, preamplifier, amplifier, multichannel analyzer and a personal computer. The spectra were collected at room temperature and under high vacuum conditions $\left(1 \cdot 10^{-7} \mathrm{mbar}\right)$. In ILEEMS a positive bias voltage is applied to the en- 
trance cone of the channeltron to enhance the detection efficiency of the resonant low-energy electrons that are more surface sensitive than the resonant conversion electrons [15]. With zero bias voltage at the cone an ILEEMS spectrum is just an ordinary Conversion Electron Mössbauer (CEMS) spectrum and, then, a deeper thickness is probed. We have recorded a polarized spectrum with a $+270 \mathrm{~V}$ positive bias voltage applied at the entrance cone of the channeltron and an unpolarized spectrum with no applied bias [16].

Analysis by micro-Raman spectroscopy was carried out with a Renishaw InVia 0310-02 system equipped with a Leica microscope and an electrically refrigerated CCD camera under continuous Nd:YAG laser excitation at $532 \mathrm{~nm}$. Low laser power outputs, in the range of $0.15-0.3 \mathrm{~mW}$, were used to prevent damage of the samples. Spectral acquisition was performed at room temperature using a focused beam on a randomly oriented sample. The diameter of the laser spot on the sample was diffraction limited by the objective lens $(\times 50)$ and calculated to be $1 \mu \mathrm{m}$. For each spectrum, 10 scans were collected and summed up over the $100-1000 \mathrm{~cm}^{-1} \mathrm{Ra}-$ man shift range to provide good signal to noise ratios. The wavenumber resolution and acquisition time were $2 \mathrm{~cm}^{-1}$ and $10 \mathrm{~s}$, respectively.

\section{Results}

The average film thickness was evaluated from various AFM profiles taken at different points of the sample. The obtained value was $1.3 \mu \mathrm{m}$. The Fe $2 \mathrm{p}$ spectrum recorded from the film (not shown) consists of a main spin orbit-doublet with binding energies (BE) of the Fe $2 p_{3 / 2}$ and $2 p_{1 / 2}$ core levels of 711.0 and 724.4 $\mathrm{eV}$, respectively. These binding energies and the presence of a small intensity shake-up satellite at $718.8 \mathrm{eV}$ are all characteristic of the presence of $\mathrm{Fe}^{3+}$ [17]. Figure 3 shows the Co $2 p$ spectrum recorded from the same sample. In this case, besides the spin-orbit doublet with BE's of $780.3 \mathrm{eV}$ (Co 2 $\mathrm{p}_{3 / 2}$ ) and $796.3 \mathrm{eV}$ (Co 2 $\mathrm{p}_{1 / 2}$ ), very intense shake-up satellite structure is observed at $786.3 \mathrm{eV}$ and $802.6 \mathrm{eV}$. These values are all fully compatible with the presence of $\mathrm{Co}^{2+}$ [4] as it should be expected for a cobalt ferrite film.

The Mössbauer spectra recorded at $300 \mathrm{~K}$ and 26 $\mathrm{K}$ from the home-made cobalt ferrite target and the commercial cobalt ferrite powder are presented in Figure 1. In both cases and at the two temperatures, the Mössbauer spectra consist of two overlapping sextets with hyperfine parameters (Table 1) which are characteristic of $\mathrm{Fe}^{3+}$ in tetrahedral and octahedral oxygen coordination and that we associate with the A and B sites, respectively, of the spinel-related structure following reported work [18].

Figure 2 shows the room temperature and $26 \mathrm{~K}$ Mössbauer spectra recorded from the PLD deposited film. The poorer signal-to-noise ratio, as compared with the powdered samples, is due to the relatively small thickness of the film. Apart from a minor central singlet in the $300 \mathrm{~K}$ spectrum which is no longer present in the $26 \mathrm{~K}$ data and that must be due to a small $\mathrm{Fe}^{3+}$ superparamagnetic component, both spectra are very similar (as they are their respective hyperfine parameters, Table 1) to those of the target and the commercial powder.

The room temperature biased and unbiased ILEEMS spectrum are presented also in Figure 2. Both spectra are very similar to that recorded in the transmission mode from the PLD film both in terms of the hyperfine parameters and the relative areas of the two sextets (Table 1). In these two spectra, however, no paramagnetic signal is observed.

In all three cases the A:B ratio obtained from the corresponding sextet areas is very different for the room temperature and the low temperature spectra. While at $300 \mathrm{~K}$ the A:B ratio is around $2.1-3.2$, at $26 \mathrm{~K}$ it is significantly lower and close to 1 . Clearly, this is a point which needs some attention and will be discussed in more detail in the discussion section.

Micro-Raman spectra of the deposits were also recorded to check the phase purity. The results are shown in Figure 4. The micro-Raman spectra of deposits and targets on $\mathrm{Si}(100)$ at $770 \mathrm{~K}$ include bands at 207, 303, 360, 470, 572, 616 and $690 \mathrm{~cm}^{-1}$ which are characteristic of cobalt ferrite $[19,20]$

The two main peaks at 470 and $690 \mathrm{~cm}^{-1}$ observed in the spectra of CFO are assigned to octahedral site (Osite) sublattice and tetrahedral site (T-site) sublattice vibration modes, respectively, and reflect the local lattice effects in the tetrahedral and octahedral sub-lattices. It is well known that the spinel structure is associated to the $\mathrm{A}_{1 g}(\mathrm{R})+\mathrm{E}_{g}(\mathrm{R})+\mathrm{T}_{1 g}+3 \mathrm{~T}_{2 g}(\mathrm{R})+2 \mathrm{~A}_{2 u}+2 \mathrm{E}_{u}$ $+4 \mathrm{~T}_{1 u}$ (IR) $+2 \mathrm{~T}_{2 u}$ phonon modes [19]. Out of these phonon modes, five are Raman active, namely $\mathrm{A}_{1 g}, \mathrm{E}_{g}$ and $3 \mathrm{~T}_{2 g}$. In the obtained spectra, the Raman modes above $600 \mathrm{~cm}^{-1}\left(616\right.$ and $\left.690 \mathrm{~cm}^{-1}\right)$ correspond to the symmetric stretching of the oxygen atom with respect to the metal-ion in tetrahedral void, tetrahedral breath mode and are assigned as $\mathrm{A}_{1 g}$. The other frequency modes below $600 \mathrm{~cm}^{-1}(207,303,360,470$ and 572 $\mathrm{cm}^{-1}$ ) correspond to the symmetric and antisymmetric bending of the oxygen atom in metal-ion (M-O) bonds at the octahedral voids and are assigned to $\mathrm{E}_{g}$ and $3 \mathrm{~T}_{2 g}$ [19]. In order to estimate the $\mathrm{Co}^{2+}$ ions ratio at the tetrahedral/octahedral sites, we calculated the intensity 


\begin{tabular}{|c|c|cccccc|}
\hline Sample & $\begin{array}{c}\mathrm{T} \\
(\mathrm{K})\end{array}$ & Sextet & $\begin{array}{c}\delta \\
\left(\mathrm{mm} \cdot \mathrm{s}^{-1}\right)\end{array}$ & $\begin{array}{c}2 \epsilon \\
\left(\mathrm{mm} \cdot \mathrm{s}^{-1}\right)\end{array}$ & $\begin{array}{c}\mathrm{H} \\
(\mathrm{T})\end{array}$ & $\begin{array}{c}\text { Area } \\
(\%)\end{array}$ & $\begin{array}{c}\text { A:B } \\
\text { ratio }\end{array}$ \\
\hline PLD target & 300 & $\mathrm{~B}$ & 0.36 & -0.04 & 52.8 & 31 & 2.2 \\
& & $\mathrm{~A}$ & 0.27 & -0.02 & 50.0 & 69 & \\
& 26 & $\mathrm{~B}$ & 0.51 & 0.02 & 55.0 & 47 & 1.1 \\
& & $\mathrm{~A}$ & 0.38 & -0.02 & 51.5 & 53 & \\
\hline Commercial & 300 & $\mathrm{~B}$ & 0.36 & -0.08 & 51.3 & 24 & 3.2 \\
target & & $\mathrm{A}$ & 0.29 & 0.00 & 48.6 & 76 & \\
& 26 & $\mathrm{~B}$ & 0.52 & -0.06 & 54.3 & 41 & 1.4 \\
& & $\mathrm{~A}$ & 0.40 & -0.02 & 51.6 & 59 & \\
\hline Film & 300 & $\mathrm{~B}$ & 0.36 & -0.02 & 51.6 & 27 & 2.5 \\
transmission & & $\mathrm{A}$ & 0.27 & 0.02 & 48.6 & 68 & \\
& & Doublet & 0.40 & 0.30 & & 5 & \\
& 26 & $\mathrm{~B}$ & 0.51 & -0.06 & 54.5 & 37 & 1.7 \\
& & $\mathrm{~A}$ & 0.37 & -0.02 & 51.1 & 63 & \\
\hline Film polarized & 300 & $\mathrm{~B}$ & 0.34 & 0.04 & 50.4 & 32 & 2.1 \\
ILEEMS & & A & 0.29 & 0.00 & 47.6 & 68 & \\
\hline Film unpolarized & 300 & B & 0.38 & -0.02 & 50.4 & 27 & 2.7 \\
ILEEMS & & A & 0.29 & -0.02 & 47.8 & 73 & \\
\hline
\end{tabular}

Table 1: Mössbauer parameters obtained from the fit of the spectra shown in Figure 1 and Figure 2

ratio of the corresponding peaks at $616 \mathrm{~cm}^{-1}$ [19] and $470 \mathrm{~cm}^{-1}[20]$. As observed in figure 4 , the peak intensity ratio increases for the films prepared by PLD which would suggest that the inversion degree in the CFO spinel-related structure might be dependent on the nature of the deposited material: thin films promote a larger inversion degree.

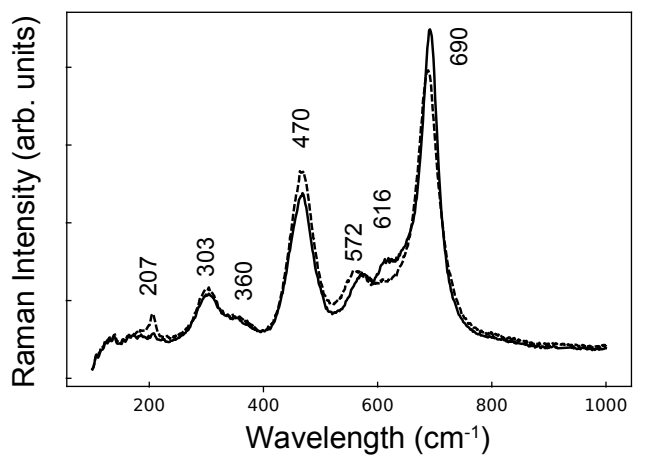

Figure 4: Micro-Raman spectra of $\mathrm{CoFe}_{2} \mathrm{O}_{4}$ target (dotted line) and PLD nanostructured films grown at 1064 on $\mathrm{Si}$ (100) substrates at 770 $\mathrm{K}$ (solid line).

\section{Discussion}

The Mössbauer and Raman spectra of the PLD film confirms that it corresponds to a genuine cobalt ferrite sample and endorse the results of our previous published work [4] on much thinner PLD films. In that case we were unable to record low temperature Mössbauer data $^{1}$ and, therefore, we were unaware of the behaviour of their corresponding Mössbauer spectra at $26 \mathrm{~K}$. The present results indicate that the method can be extended to produce much thicker films, to the point that it is possible to record transmission Mössbauer spectra, without loss of the structural and compositional quality of the films. The trend observed both in the dramatic change in the A:B sextets area ratio and the evolution of the hyperfine parameters with decreasing temperature is comparable to that observed in the powdered samples.

Comparison of the ILEEMS and transmission spectra recorded from the PLD film do not show significant, striking differences. Their respective hyperfine parameters are basically the same (within the error of the experimental determination) indicating that the surface region retains the spinel structure of the bulk. The A:B sextet ratio shows small variations: 2.5 for the transmission spectrum, 2.1 for the biased ILEEMS spectrum and 2.7 for the unbiased ILEEMS spectrum. The A:B ratio in the target used to produce the films is 2.2, i.e., very close to that observed in the polarized ILEEMS spectrum. Given that the signal-to-noise ratio of this spectrum is the best of the three spectra recorded from the

\footnotetext{
${ }^{1}$ Those films were measured with a room temperature CEMS (conversion electron Mössbauer spectroscopy) setup.
} 
film, we think that the variations observed in this ratio between these three spectra is mainly related to the large uncertainty in the determination of the sextets area in the transmission and unbiased ILEEMS spectra which are quite noisy. Given the different surface sensitivities of the various Mössbauer spectra, the results would indicate that the composition of the PLD film is quite homogeneous in all its thickness except for the presence of the superparamagnetic component. Since this is absent in both ILEEMS spectra but it is observed in the transmission spectrum (that probes the complete thickness of the film) it must be located at the interphase of the PLD film and the Si wafer. In any case, it contributes only about a $5 \%$ of the total film.

This homogeneous composition with depth is quite significant and can have serious implications when seeking a possible application for these films. In a previous work [15], we have found comparing ILEEMS, CEMS and transmission spectra recorded from acicular $\mathrm{Fe}_{2} \mathrm{O}_{3}$ nanoparticles (which also have a spinel related structure) very large variations (close to $100 \%$ ) of the A:B sextet ratio that suggested an inhomogeneous cation distribution from the surface layers to the nanoparticles bulk. While in these nanoparticles surface effects were clearly evident, probably due to their characteristic shape, the present results indicate that cobalt ferrite films of micrometer thicknesses can be produced by PLD having a relatively homogeneous cation distribution from the surface layers to the bulk.

The large decrease in A:B sextet area ratio with temperature in the Mössbauer spectra is also an intriguing fact that deserves some comment, as this ratio is commonly used to determine the inversion degree of cobalt ferrite. If this ratio changes with temperature, the inversion degree should, in a first, crude approximation, change with temperature and, consequently, an explanation is needed.

It can be argued that because the strong overlap of the two sextets, the area ratio obtained, particularly at room temperature, is not reliable, as it depends on the constraints imposed on the fitting. It is undeniable that the area ratio obtained from the fit of the spectra at room temperature is highly dependent, for example, on the linewidth of the two sextets. This problem is much reduced, however, at $26 \mathrm{~K}$, as the different variation with temperature of the isomer shifts and hyperfine magnetic fields of both sextets, together with the narrowing of the spectral lines, result in a much smaller overlap which is better resolved when fitting the data. Inspection of the shape of the high velocity lines in the room and low temperature spectra (around $9.5-10 \mathrm{~mm} \cdot \mathrm{s}^{-1}$ ) clearly indicates that, although difficult of quantify, this variation exists as the depth of the line corresponding to the octahedral site is significantly larger, in all the cases presented in the paper, in the $26 \mathrm{~K}$ spectrum.

It is well-known that the cation distribution in cobalt ferrite depends on the preparation temperature and particular thermal history of a given sample [21]. More specifically, it is affected by the cooling rate from the high temperature of the last step of the synthesis. At elevated temperatures the cations present a high mobility between the different sites in the spinel related structure and a particular cation distribution can be obtained by freezing it using a specific cooling rate. In any case, there is a limit temperature, which is situated much more above room temperature, from which the cation distribution does not change any longer with decreasing temperature. This temperature is related to the diffusion barriers for cations in the spinel structure $[22,23]$. Based on this last fact, and taking into account that well below room temperature any cation mobility is very limited, we do not think that the decrease in A:B ratio from $300 \mathrm{~K}$ to $26 \mathrm{~K}$ can be due to a change in the cation distribution.

It has been reported [24, 25] that this change can be due to different recoil free fractions (or, alternatively, to different Debye temperatures) of the tetrahedral and octahedral sublattices. In a recent paper [12] we have estimated the required Debye temperatures associated to the two lattices to observe a variation as that reported in the present work. According to our calculations, that basically agree with previously reported data by other authors [24, 25], both Debye temperatures should be extremely different: $735 \mathrm{~K}$ for the tetrahedral sublattice and $195-235 \mathrm{~K}$ for the octahedral lattice. This tremendous variation in Debye temperatures is rather unusual. Although it is known that they can be different for both sublattices [18], this difference is usually not so extreme. For example in magnetite they are $334 \mathrm{~K}$ and $314 \mathrm{~K}$ for A and B sites, respectively. This dissimilarity in Debye temperatures would have significant structural implications and explaining such difference in the different rigidity of the two sublattices is of difficult rationalization. Therefore, although we think plausible that some variation is present in the recoil free fractions of the A and B sites, as it is usually found in spinel oxide compounds, we do not believe that the behaviour observed in the Mössbauer spectra can be solely assigned to the difference in Debye temperatures. In view of our EXAFS results, which will be the subject of a subsequent publication, this is not occurring.

Another fact that must not be disregarded is the ocurrence of supertransferred magnetic fields in the spinel structure. As pointed out by Vandenberghe and De 
Grave [18], due to the supertransfer mechanism, a significant percentage of the $\mathrm{Fe}^{3+}$ at the octahedral sites experience hyperfine magnetic fields which can be very similar and even smaller than the average hyperfine field felt by the tetrahedral $\mathrm{Fe}^{3+}$ cations. Given that this is one of the Mössbauer parameters that is used to identify the iron cations in octahedral and tetrahedral sites, the effect would lead to an overestimate of the area of the sextet corresponding to the A sites. This can explain to a large extent the very large $\mathrm{A}: \mathrm{B}$ ratio observed in the room temperature spectrum. An A:B area ratio between 2.0 and 3.0 as is not compatible with a formulation of $\mathrm{CoFe}_{2} \mathrm{O}_{4}$ neither as a direct nor as completely inverse spinel.

As mentioned above, as temperature decreases, the different variation of the isomer shifts and hyperfine magnetic fields of the $\mathrm{A}$ and $\mathrm{B}$ cations diminish the overlap between the two sextets and the A:B ratio becomes more realistic. However, at low temperature the ratio between the two sextets A:B is still typically larger than 1.0. This indicates that even then, the occurrence of supertransferred magnetic fields might still play a relevant role.

It follows from the above that there is a multiplicity of concomitant aspects that could explain the values observed for the A:B sextet ratios: the inherent difficulty in fitting with only two Lorentzian-shaped sextets strongly overlapping subspectra, the differences in the recoilfree fractions of the A and B sublattices and the occurrence of supertransferred hyperfine magnetic fields. As a consequence, it seems that zero-field Mössbauer spectroscopy might not be specially suited to calculate the inversion degree in CFO systems and that the combination of Mössbauer spectroscopy under high applied magnetic fields with some other structural characterization tools (such as EXAFS) would be more appropriate for this task. Work is in progress to explore this approach.

\section{Summary}

We have grown by pulsed-laser deposition at 1064 $\mathrm{nm}$ a micrometer thick film of $\mathrm{CoFe}_{2} \mathrm{O}_{4}$. The transmission Mössbauer spectra for both the film and the targets have been measured at room temperature and low temperature $(26 \mathrm{~K})$. The spectra have been fitted by two sextets, which have been correlated with $\mathrm{Fe}^{3+}$ in tetrahedral and octahedral sites. Surface-sensitive Mössbauer spectra recorded from the PLD film did not show significant differences with the bulk characteristic Mössbauer data suggesting an homogeneous cation distribution of the film all along its thickness. However, there is a large variation in the area ratio of the two sextets with decreasing temperature. This effect, which has been previously reported for targets, is now reported, for the first time, also for films. The detailed origin of the observed ratios is likely due to a combination of several effects and complicates the determination of the tetrahedral and octahedral populations, and then of the inversion degree, in spinel ferrites.

\section{Acknowledgments}

This research was supported by the Spanish Ministry of Economy and Competitiveness (MINECO) through Projects CTQ2016-75880-P and MAT2015-64110-C2$1-\mathrm{P}$ and by the European Commission through the project AMPHIBIAN H2020-NMBP-2016-720853.

\section{References}

\section{References}

[1] M. Lelis, A. Porto, C. Gonçalves, J. Fabris, Cation occupancy sites in synthetic Co-doped magnetites as determined with Xray absorption (XAS) and Mössbauer spectroscopies, J. Mag. Mag. Mat. 278 (2004) 263-269.

[2] M. Sugimoto, The past, present, and future of ferrites, J. Amer. Ceram. Soc. 82 (2004) 269-280.

[3] M. Kaur, N. Kaur, V. , Ferrites: Synthesis and applications for environmental remediation, in: Ferrites and Ferrates: Chemistry and Applications in Sustainable Energy and Environmental Remediation, volume 1238 of ACS Symposium Series, American Chemical Society, 2016, pp. 113-136.

[4] M. Oujja, L. Martín-García, E. Rebollar, A. Quesada, M. A. García, J. F. Fernández, J. F. Marco, J. de la Figuera, M. Castillejo, Effect of wavelength, deposition temperature and substrate type on cobalt ferrite thin films grown by pulsed laser deposition, App. Surf. Sci. 452 (2018) 19-31.

[5] Y. H. Hou, Y. J. Zhao, Z. W. Liu, H. Y. Yu, X. C. Zhong, W. Q. Qiu, D. C. Zeng, L. S. Wen, Structural, electronic and magnetic properties of partially inverse spinel $\mathrm{CoFe}_{2} \mathrm{O}_{4}$ : a firstprinciples study, J. Phys. D: App. Phys. 43 (2010) 445003.

[6] G. Dascalu, O. F. Caltun, $\mathrm{CoFe}_{2} \mathrm{O}_{4}$ thin films deposited by PLD with in situ heating and post annealing, J. Opto. Adv. Mat. 13 (2011) 1145-1148.

[7] G. B. Alcantara, L. G. Paterno, F. J. Fonseca, M. A. Pereirada Silva, P. C. Morais, M. A. G. Soler, Dielectric properties of cobalt ferrite nanoparticles in ultrathin nanocomposite films, Phys. Chem. Chem. Phys. 15 (2013) 19853-19861.

[8] D. Mukherjee, M. Hordagoda, R. Hyde, N. Bingham, H. Srikanth, S. Witanachchi, P. Mukherjee, Nanocolumnar Interfaces and Enhanced Magnetic Coercivity in Preferentially oriented Cobalt Ferrite Thin Films Grown Using Oblique-Angle Pulsed Laser Deposition, ACS Appl. Mater. Interfaces 5 (2013) 7450-7457.

[9] S. D. Sartale, C. D. Lokhande, V. Ganesan, Electrochemical deposition and characterization of $\mathrm{CoFe}_{2} \mathrm{O}_{4}$ thin films, Phys. Stat. Sol. (a) 202 (2005) 85-94.

[10] W. Rao, Y. B. Wang, Y. A. Wang, J. X. Gao, W. L. Zhou, J. Yu, Surface morphology and magnetic properties of $\mathrm{CoFe}_{2} \mathrm{O}_{4}$ thin films prepared via sol-gel method, Adv. Mater. Res. 752-754 (2013) 1024-1028. 
[11] F. W. Oliver, D. Seifu, E. J. Hoffman, V. Webb, Transmission Mössbauer Spectroscopy on Pulsed-Laser-Deposited Thick CoFe2o4 Films, Hyperfine Interact. 153 (2004) 57-60.

[12] J. de la Figuera, A. Quesada, L. Martín-García, M. Sanz, M. Oujja, M. Castillejo, A. Mascaraque, A. T. N'Diaye, M. Foerster, L. Aballe, J. F. Marco, Mössbauer and Magnetic Properties of Coherently Mixed Magnetite-Cobalt Ferrite Grown by Infrared Pulsed-Laser Deposition, Croatica Chem. Acta 88 (2015) 453460.

[13] J. de la Figuera, A. Quesada, L. Martín-García, M. Sanz, M. Oujja, E. Rebollar, M. Castillejo, P. Prieto, A. Muñoz-Martín, L. Aballe, J. F. Marco, Self-organized single crystal mixed magnetite/cobalt ferrite films grown by infrared pulsed-laser deposition, App. Surf. Sci. 359 (2015) 480-485.

[14] J. Huotari, V. Kekkonen, T. Haapalainen, M. Leidinger, T. Sauerwald, J. Puustinen, J. Liimatainen, J. Lappalainen, Pulsed laser deposition of metal oxide nanostructures for highly sensitive gas sensor applications, Sens. Act. B: Chem. 236 (2016) 978-987.

[15] J. Marco, J. Gancedo, M. Monti, J. de la Figuera, Mössbauer spectroscopy and surface analysis, in: G. K. y. T. N. V.K. Sharma (Ed.), Mössbauer Spectroscopy: Applications in Chemistry, Biology and Nanotechnology, John Wiley \& Sons, New Jersey, 2013, pp. 455-469.

[16] F. Moutinho, C. Rojas, L. D’Onofrio, Design of a conversion electron Mössbauer spectrometer based on an electron multiplier. Evaluation of the mean-escape-depth of the detected signals, in: J. Desimoni, C. Ramos, B. Arcondo, F. D. Saccone, R. Mercader (Eds.), LACAME 2008, Springer Berlin Heidelberg, 2009, pp. 141-147.

[17] P. Prieto, J. F. Marco, J. E. Prieto, S. Ruiz-Gomez, L. Perez, R. P. del Real, M. Vázquez, J. de la Figuera, Epitaxial integration of $\mathrm{CoFe} 204$ thin films on $\mathrm{Si}$ (001) surfaces using TiN buffer layers, App. Surf. Sci. 436 (2018) 1067-1074.

[18] R. E. Vandenberghe, E. D. Grave, Mössbauer effect studies of oxidic spinels, in: G. J. Long, F. Grandjean (Eds.), Mössbauer Spectroscopy Applied to Inorganic Chemistry, number 3 in Modern Inorganic Chemistry, Springer US, 1989, pp. 59-182.

[19] P. Chandramohan, M. P. Srinivasan, S. Velmurugan, S. V. Narasimhan, Cation distribution and particle size effect on Raman spectrum of $\mathrm{CoFe}_{2} \mathrm{O}_{4}$, J. Sol. Stat. Chem. 184 (2011) 8996.

[20] X. Fan, J. Guan, X. Cao, W. Wang, F. Mou, Low-temperature synthesis, magnetic and microwave electromagnetic properties of substoichiometric spinel cobalt ferrite octahedra, Euro. J. Inorg. Chem. 2010 (2010) 419-426.

[21] M. Takahashi, M. E. Fine, Magnetic behavior of quenched and aged $\mathrm{CoFe}_{2} \mathrm{O} 4-\mathrm{Co}_{3} \mathrm{O}_{4}$ alloys, J. App. Phys. 43 (1972) 42054216.

[22] R. Dieckmann, H. Schmalzried, Defects and cation diffusion in magnetite (II), Berichte Bunseng. Physik. Chemie 81 (1977) 414-419.

[23] C. L. Muhich, V. J. Aston, R. M. Trottier, A. W. Weimer, C. B. Musgrave, First-principles analysis of cation diffusion in mixed metal ferrite spinels, Chem. Mat. 28 (2016) 214-226.

[24] S. J. Kim, S. W. Lee, S. Y. An, C. S. Kim, Mössbauer studies of superexchange interactions and atomic migration in $\mathrm{CoFe}_{2} \mathrm{O}_{4}$, J. Mag. Mag. Mat. 215-216 (2000) 210-212.

[25] S. J. Kim, S. W. Lee, C. S. Kim, Mössbauer studies on exchange interactions in $\mathrm{CoFe}_{2} \mathrm{O}_{4}$, Jap. J. App. Phys. 40 (2001) 4897. 